- it seems that no structural analyses of carried out. The failure to retain thermal polymers, and the absence of data on the retention of protein and nucleic acids, leads me to the opinion that microspheres formed from thermal polymers could not have served to encapsulate the first life on the primitive Earth.

Because these fundamental proposals have such poor experimental support, I am forced to conclude that Fox's theory has little validity. Consequently, the other claims to be found in the book - on the spheres, their implied sexuality, the 'membrane' potentials and so on - are meaningless in the context of the origins of life. This is not to say that polypeptides did not have a role in the first life, or that there might have been some sequences that formed more readily than others, but it does not seem likely that proteins with the presumed boundary material were significance of the budding of micro-

specified sequences formed without some sort of a catalyst (code?) to direct their formation. Investigation of syntheses using conditions that are more selective than dry, thermal polymerization may shed some light on prebiotic peptide formation.

On the positive side, the book provides a lively account of Fox's ideas and of how they developed. Fox is a strong proponent of the view that the most useful insights for the understanding of the origin of life come from knowledge of the products of potentially prebiotic precursors and not from the examination of contemporary and ancient biochemical systems; that is a view that is gaining wider acceptance today. But most of the conclusions that he has drawn far exceed what is justified by the data.

James P. Ferris is a Professor in the Department of Chemistry, Rensselaer Polytechnic Institute, Troy, New York 12180, USA.

\section{Set and match}

\section{Ian Stewart}

Fractals Everywhere. By Michael Barnsley. Academic: 1988. Pp. 396. £27, \$39.95.

Noт too long ago there was only one book on fractals, Benoit Mandelbrot's The Fractal Geometry of Nature. Now there are several, but here we have the first genuine textbook on fractals as fractals (the admirable Geometry of Fractal Sets, by Ken Falconer, is too much a textbook on geometric measure theory to count here). Michael Barnsley has taught the material at the Georgia Institute of Technology, where his course is open to all students who have completed two years of calculus; the resulting volume could be used by final-year undergraduates or at postgraduate level.

Barnsley concentrates on the mathematics that underlies the theory of fractals: the only application discussed is to data-compression in computer graphics. He takes pains to convey the ideas behind the theorems, using diagrams as well as formal definitions to put across abstract concepts such as metric space, affine transformation and Hausdorff dimension.

The mathematical core of the book is the notion of a self-similar set, which is fundamental to the fractal point of view. A set is self-similar if there exists some finite system of transformations - or iterated function system - such that the images of the set under these transformations, when combined, yield the original set. In other words, the set can be split into a finite number of pieces, each of which has the same structure as the whole, but on a smaller scale. Every iterated function system possesses a unique invariant set - that is, a set which is self-similar under that system. This invariant set is in general a fractal, except for unusual choices of the function system.

One of the central results concerns the 'chaos game'. If an initial point is chosen, and transformations from the function system are applied to it at random to create new points, then with probability one the set of points so formed becomes closer and closer to the unique invariant set for the system. This forms the basis of a computer algorithm for drawing the invariant set. Barnsley deals in depth with the mathematical and dynamical features of the chaos game (including the fractal dimension of the invariant set), alongside its application to computer graphics. The ideas are also extended to a probabilistic version, in which the black-and-white decision 'point' or 'no point' is replaced by shading on a scale of grey. The mathematics behind the famous Mandelbrot set, and its associated Julia sets, is discussed in some detail.

This is a fetching book, with beautiful computer graphics (some in colour), as well as a host of more schematic diagrams, the aim of which is to clarify the abstract mathematics. The fractal ferns, in particular, are quite remarkable, and relate directly to the central ideas. The material is well chosen and hangs together coherently, and the writing conveys a proper sense of excitement. It is true that some of the proofs are tough going, and demand a certain mathematical maturity; but the book can be understood even if the occasional proof is set to one side for future investigation. If you want a genuine understanding of fractals as mathematics, and not just as attractive images, this is an excellent place from which to start.

Ian Stewart is in the Mathematics Institute, University of Warwick, Coventry CV4 7AL, UK.

\section{Innervative ideas}

\section{Alun M. Davies}

Body and Brain: A Trophic Theory of Neural Connections. By Dale Purves. Harvard University Press: 1988. Pp.321. $\$ 35, £ 27.95$.

IT Is common knowledge that the brain controls the body. That the body has an organizing influence on the nervous system is not generally appreciated, yet maturation and growth in all but the simplest of organisms is accompanied by the continuous adaptation of neural connections to the body's changing needs. Purves takes this adaptation as the starting point for the exposition of a trophic theory of neural connectivity. The theory not only provides an important unifying principle in neural development, but has implications for several diverse areas of biological science.

In the context of the nervous system, the term trophic refers to the long-term interdependence between neurons and the cells they innervate. In development, these trophic interactions regulate the size of neuronal populations and have a part in modifying the extent and complexity of neural connections throughout life. Purves presents a comprehensive overview of the foundations of trophic theory from its origins in experimental embryology to our current understanding of its molecular basis.

Alongside the central theme of trophic interactions in development and growth, there is discussion, from an evolutionary standpoint, of the significance of the adaptability of the nervous system to changes in body size and form. The link between neural activity and the modification of connections is examined in terms of trophic theory, an issue which has a bearing on the molecular basis of learning. Finally, Purves evaluates the currently fashionable regressive theories of neural connectivity that are based on a direct analogy between neural development and natural selection. His arguments clearly expose the inadequacies of the naive view that the nervous system develops according to some abstract darwinian principle.

This is a scholarly, thoughtful and wellwritten book. It will be of value not only to neurobiologists but to anyone interested in basic biological issues.

Alun M. Davies is Senior Lecturer in Anatomy at St George's Hospital Medical School, Tooting, London SW17 ORE, UK

\section{New in paperback}

Cerebral Dominance: the Biological Foundations edited by Norman Geschwind and Albert M. Galaburda. Publisher is Harvard University Press, price is $£ 10.50, \$ 12.95$. For review see Nature 314, 475 (1985 